Classification

Physics Abstracts

$64.70 \mathrm{~J}-68.10 \mathrm{C}-82.70$

\title{
Anomalous viscosity of microemulsions near a critical point
}

\author{
A. M. Cazabat, D. Langevin and O. Sorba \\ Laboratoire de Spectroscopie Hertzienne de l'ENS, 24, rue Lhomond, 75231 Paris Cedex 05, France
}

(Reçu le 12 mars 1982, accepté le 18 mai 1982)

\begin{abstract}
Résumé. - Nous avons observé une anomalie de la viscosité de cisaillement de phases microémulsions au voisinage d'un point critique. Malgré un bon accord qualitatif avec la théorie, l'interprétation quantitative est encore insuffisante.
\end{abstract}

\begin{abstract}
We have observed an anomalous behaviour of the shear viscosity close to a critical point in microemulsion systems. Although there is a good qualitative agreement between experiments and theory, the quantitative comparison is less satisfactory.
\end{abstract}

1. Introduction - An anomalous increase of the shear viscosity $\eta$ has been experimentally observed in many binary mixtures as well as in ternary mixtures near their critical consolute point $[1,2]$. It was early hypothesized that the anomalous viscosity must arise from the anomalous increase in the concentration fluctuations which is also responsible for the anomalies observed in various other transport properties near the critical point [3]. The recent refinement of modecoupling theory and the extension of renormalization group techniques to the treatment of dynamic critical phenomena have enabled the critical exponent $y_{\eta}$ for the shear viscosity [4] to be calculated. A common feature of all these calculations is that $y_{\eta}$ is small $\left(y_{\eta} \sim 0.04\right)$ and that the divergence of $\eta$ is weak as confirmed by the experiments.

Critical behaviour has also been reported recently in colloidal dispersions : protein solutions [5], micellar solutions [6], microemulsions [7-11]. These systems are mixtures of entities of unequal size : solvent molecules (size $a$ ) and macromolecules or surfactant molecules (size $l>a$ ). The critical behaviour of both protein and micellar solutions differs from that of simpler binary mixtures in that the correlation length of the concentration fluctuations $\xi$ diverges with the mean field theory critical exponent $v=0.5$ (instead of $v \sim 0.63$ ), and its scale factor $\xi_{0}$ is of the order of $l$. An extremely large viscosity anomaly has been found in protein solutions $\left(y_{\eta} \sim 1\right)$, but no noticeable anomaly was visible in surfactant aqueous solutions $\left(y_{\eta}<0.02\right)$.

Microemulsions are dispersions of water (or oil) droplets surrounded by surfactant molecules in a continuous organic (or aqueous) medium. The droplet size is small : 50-200 $\AA$. Besides its intrinsic interest, the study of phase transitions in microemulsions is relevant for many practical applications such as oil recovery [12]. The critical behaviour of microemulsions is not yet well understood. The critical exponents are not the mean field values and $\xi_{0}$ is intermediate between $a$ and $l$. But the reported values of $v$ are somewhat dispersed (0.68 to 1.13 ) [9-10].

We present here a study of the anomalous viscosity of microemulsions near a critical point. Viscosity measurements will be analysed by comparison with correlation length values as deduced from the angular variation of the scattered light intensity. 
2. Theoretical background. - The correlation length of the concentration fluctuations in a multicomponent system diverges at the critical point according to [13]

$$
\xi=\xi_{0} \varepsilon^{v}
$$

where $\varepsilon=\left|\frac{T-T_{\mathrm{c}}}{T_{\mathrm{c}}}\right|$ or $\left|\frac{\mu_{i}-\mu_{i \mathrm{c}}}{\mu_{\mathrm{ic}}}\right|, T$ being the temperature, $\mu_{i}$ the chemical potential of component $i$ and $T_{\mathrm{c}}$ and $\mu_{\mathrm{ic}}$ their values at the critical point. The exponent $v$ depends on the path used to approach the critical point (temperature fixed, or concentration of component $i$ fixed), but remains the same along the paths asymptotically parallel to the coexistence surface in the phase diagram [14].

The increase in the concentration fluctuations gives rise to a strong scattering of light close to the critical point. The angular variation of the scattered intensity can be represented by the Orstein-Zernike formula [13].

$$
I(q)=I(0) /\left(1+q^{2} \xi^{2}\right)
$$

with $q=(4 \pi n / \lambda) \sin \theta / 2, n$ being the refractive index of the solution, $\lambda$ the wavelength of light in vacuo and $\theta$ the scattering angle.

Moreover

$$
I(0) \sim \varepsilon^{\gamma} \quad \gamma=2 v .
$$

The earliest mean field and mode-coupling theories of the anomalous viscosity led to an increase of the viscosity due to the concentration fluctuations of the form [15] :

$$
\Delta \eta=\eta-\eta_{0}=k_{\mathrm{B}} T / 160 \pi \xi D(q=0)
$$

$D(q)$ being the $q$ dependent diffusion coefficient for the Fourier components of wave vector $q$ of concentration fluctuations. As $D(q=0)=k_{\mathrm{B}} T / 6 \pi \eta \xi$, no anomalous viscosity is expected from this theory :

$$
\eta=\eta_{0}+3 \eta / 80=\eta_{\text {reg }} .
$$

The more recent theories predict either logarithmic, or power law divergences. They can be regarded as a more accurate treatment of the pair correlation function leading to a modified Orstein-Zernike formula [4]

and

$$
\begin{gathered}
I(q)=I(0) /\left(1+q^{2} \xi^{2}\right)^{1-\mu / 2} \\
\gamma=(2-\mu) \nu .
\end{gathered}
$$

Provided the critical point is not too close, the logarithmic and power law formulae for the viscosity are equivalent, and $\eta$ can be written as [16-18] :

$$
\eta=\eta_{\text {reg }}\left(Q_{0} \xi\right)^{x_{n}} \sim \varepsilon^{y_{n}}
$$

$Q_{0}$ being an adjustable parameter and $x_{\eta}=y_{\eta} / v$.

The critical exponents $\mu$ and $y_{\eta}$ are related through :

$$
y_{\eta}=v-y_{A}-\mu v
$$

$y_{A}$ being the critical exponent for the mass conductivity; $y_{A}$ is close to $v$ and both $\mu$ and $y_{\eta}$ are small.

From mode-coupling theories $x_{\eta}$ is found to be 0.054 using the self consistent approximation and 0.070 with vertex corrections. The renormalization group theory predicts $x_{\eta}$ to be 0.053 
in a first order expansion and 0.065 in a second order expansion in three dimensions. These calculations are in good agreement with the experiments on binary and ternary mixtures : $0.065<x_{\eta}<0.070$ [2].

3. Experimental results. - The samples are quaternary mixtures of water (bidistilled), sodium dodecyl sulfate (SDS, BDH product), toluene and n-butanol or n-pentanol (Merck products, pure for analysis).

The experimental set up for light scattering measurements has already been described elsewhere [7]. The shear viscosities are measured with an Ubbelhode flow viscometer. The shear rates have been varied between 100 and $200 \mathrm{~s}^{-1}$. As expected, the viscosity values were not affected, since these shear rates are small compared to the inverse correlation time of the concentration fluctuations $\tau^{-1}[18]$ :

$$
\frac{1}{\tau}=\frac{k_{\mathrm{B}} T}{6 \pi \eta \xi^{3}} \sim 4 \times 10^{3} \mathrm{~s}^{-1}
$$

in our experiments.

The temperature of the samples is controlled within $\pm 0.05^{\circ} \mathrm{C}$ by the means of a liquid water bath (Haake E3-V).

Different series of samples have been prepared in order to approach the critical point along different paths.

3.1 COMPOSITION VARIATION AT CONSTANT TEMPERATURE $T=20^{\circ} \mathrm{C}$. - We have fixed the water/soap ratio to a constant value $: 1.25 \mathrm{~cm}^{3}$ of water per gram of SDS in the droplets. This allows us to keep constant the radius $R$ of the microemulsions droplets along the path following the coexistence surface (see Fig. 1). (This path is linear (dilution line) for volume fractions $\phi$ less than 0.3. For higher volume fractions the curvature of the path indicates some structural changes in the microemulsion related to the inversion process.) Light scattering experiments already indicated that $R=41 \AA$ for the system with n-butanol, and $R=45 \AA$ with n-pentanol [7]. The variation of the shear viscosity along this path is represented on figure 2 , for the two microemulsion series. The viscosity anomaly of the microemulsions with butanol around a droplet volume fraction $\phi \sim 10 \%$ is associated with a steep increase of the electrical conductivity and a more progressive but strong increase of the sample turbidity. All these features are absent in the microemulsions with pentanol [7]. The increase of electrical conductivity has been associated with a percolation

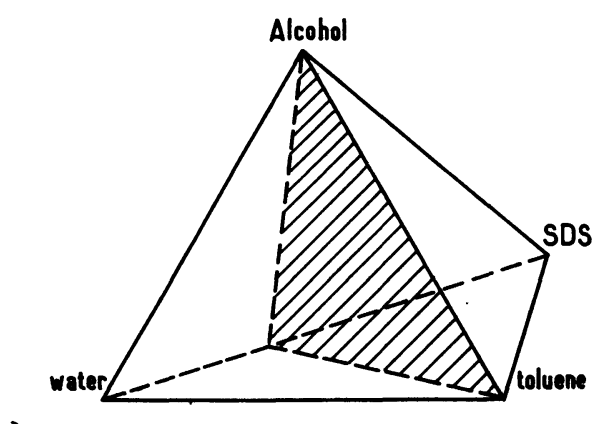

a)

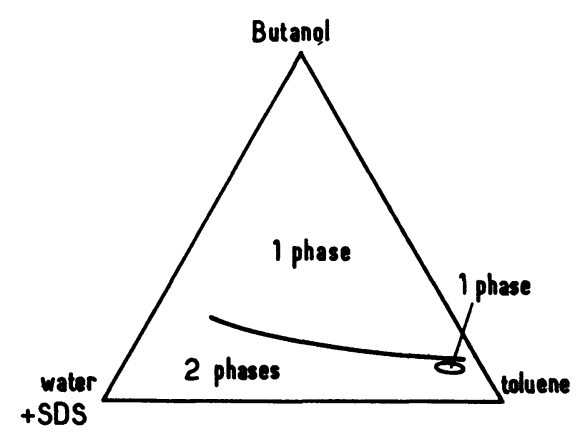

b)

Fig. 1. - a) Schematic quaternary phase diagram; the dashed triangle represents the plane of study. b) Pseudo ternary phase diagram of the system water-toluene-butanol-SDS in which we have represented the demixion line and the « birefringent microemulsions " lobe. Stars correspond to « birefringent microemulsions". 


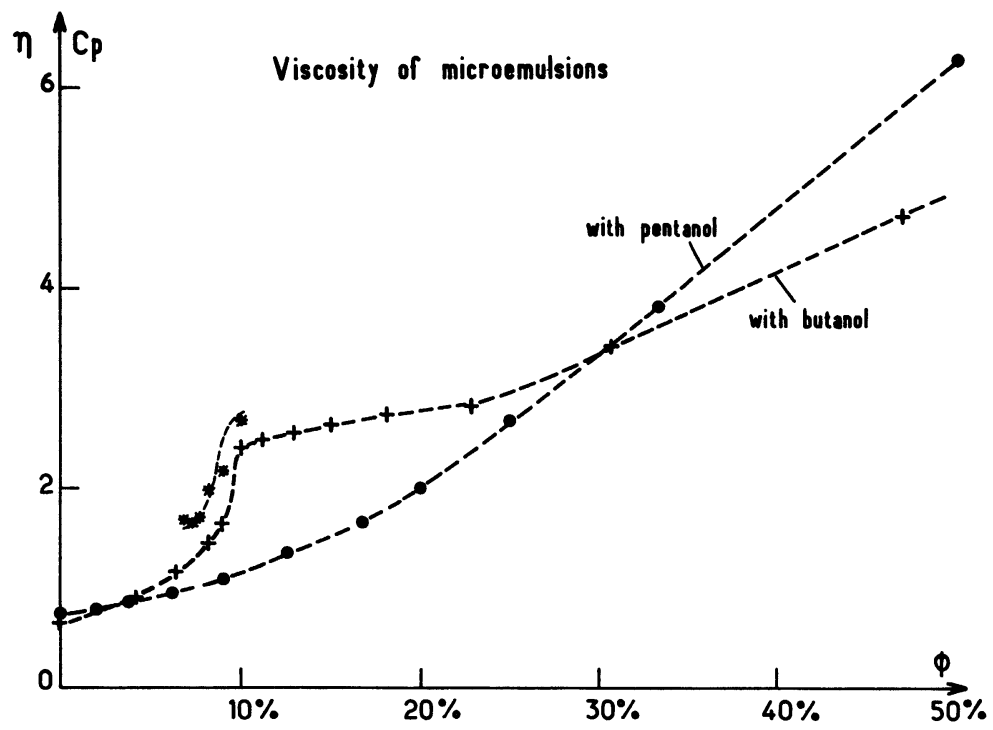

Fig. 2. - Variation of the viscosity of microemulsion with butanol and pentanol versus droplets volume fraction $\phi$ along the demixion line. The demixion curve in the system water-toluene-pentanol-SDS is slightly upwards ( $40 \%$ more pentanol than butanol). There is no lobe in this case.

phenomena, whereas the light scattering features are more likely to be due to the vicinity of a critical point [7].

There still is no satisfactory explanation of this apparently fortuitous relation between the percolation and critical points.

In order to characterize the distance $\left|\left(\mu_{i}-\mu_{i \mathrm{c}}\right) / \mu_{i \mathrm{c}}\right|$ from the critical point, we tried to study the two phase region of the phase diagram, by varying the alcohol concentration (Fig. 1). This attempt failed, because very close to the demixion line for $\phi \sim 10 \%$ the two phase region was very small and bounded by a monophasic region of "birefringent microemulsions ". These special features seem to be commonly associated with the vicinity of critical points in water in oil microemulsion without salt [19].

The " birefringent microemulsions " are thought to be very dilute lamellar systems [20]. In order to verify that the anomalous viscosity was not due to precursor fluctuations of a lamellar structure, we have also measured the viscosity of these systems along the upper border line of the lobe of figure 1 . The measurements are reported on figure 2 . It can be noted that : the shear viscosities of these phases are not much larger than for the non birefringent microemulsions $\left(^{1}\right.$ ) and the two viscosity curves are parallel indicating a similar pre-critical behaviour.

3.2 TEMPERATURE VARIATION AT CONSTANT COMPOSITION. - We have made another attempt to characterize the distance $t$ to the critical point by varying the temperature $\left(\varepsilon=\left|T-T_{\mathrm{c}}\right| / T_{\mathrm{c}}\right)$. This attempt was not more satisfactory : except for the most dilute samples $(\phi<0.08)$ above $20^{\circ} \mathrm{C}$ where a phase separation occurs, the demixion line of figure 1 moves downwards; the microemulsions are no longer at the edge of the monophasic region, and the path followed in this way is not contained on the coexistence surface. The meaning of the theoretical expressions of $\S 2$ is therefore questionable along this path.

( ${ }^{1}$ ) The viscosities of the birefringent microemulsions of reference [20] are much larger, but their alcohol content is much lower. 
In all cases the samples closest to the critical point were those corresponding to $T=20^{\circ} \mathrm{C}$ as evidenced from the correlation length variation with temperature represented on figure 3 .

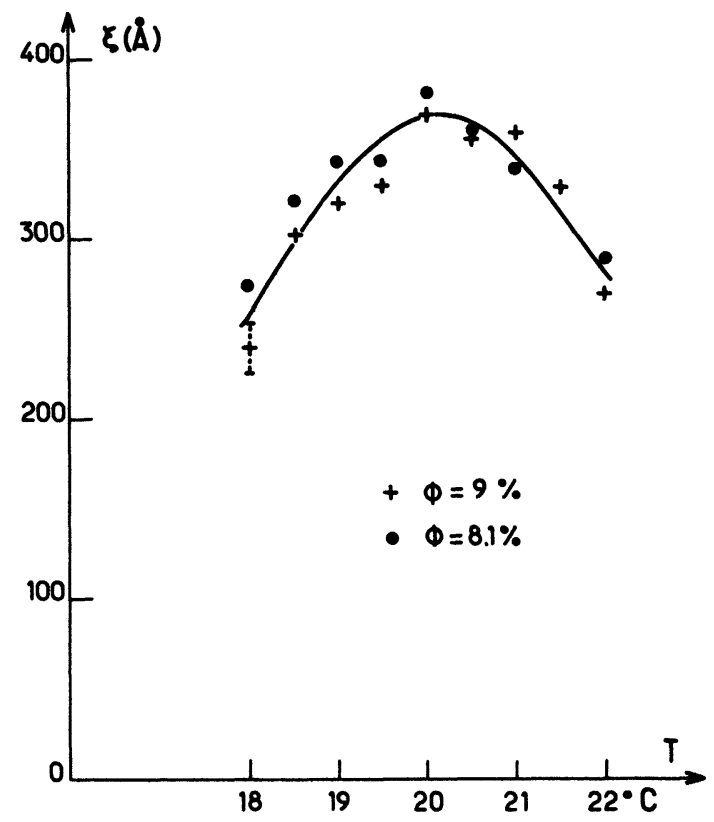

Fig. 3. - Variation of the correlation length $\xi$ versus temperature for the butanol-microemulsions closest to the critical point.

The corresponding viscosity measurements revealed no significant variations $\left({ }^{2}\right)$ between $20^{\circ}$ and $18{ }^{\circ} \mathrm{C}$. As the correlation length is decreasing, we interpreted this result by an increase in $\eta_{\text {reg }}$ that compensates the decrease of the factor $\left(Q_{0} \xi\right)^{x_{n}}$ in equation (5).

4. Discussion - Since both $\mu$ and $\xi$ are unknown, but since $\mu$ is expected to be small, we have first tried to fit our light scattering data with the Orstein-Zernike approximate formula (2). The fits are quite satisfactory in the whole angular range $10<\theta<160^{\circ}$ for the studied microemulsions.

Since the experimental intensity measurement accuracy was better than $3 \%$ and the measured $\xi$ less than $500 \AA, \mu$ is less than 0.2 . Let us recall that theory predicts $\mu=0.05$. In order to confirm this result we have represented on figure 4 the variation of $I(q=0)$ with $\xi$ in a $\log -\log$ plot. This gives : $\gamma / v=2 \pm 0.2$ consistent with $\mu<0.2$. Improvements of the experimental set up are currently under way to improve the determination of $\mu$.

We have represented in figure 5 a tentative fit of viscosity data with equation (5). The regular part of the viscosity has been chosen close to those for pentanol microemulsions. The anomalous part variation with volume fraction is very asymmetrical with respect to $\phi \sim 0.1$, in contrast to the behaviour of the correlation lengths. Two extreme $Q_{0}$ values have therefore been used. In

(2) Within experimental reproducibility : better than $1 \%$ for the non critical samples, and around $5 \%$ for the most turbid samples for which a better temperature control is required to improve the reproducibility. 


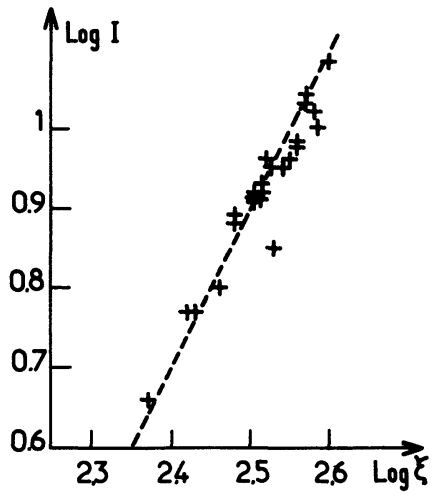

Fig. 4. - Log-log plot of the extrapolated scattered light intensity to zero scattering wave vector versus correlation length. The line represents the best fit with the slope $\gamma / v=2 . I$ : arbitrary units; $\xi: \AA$.

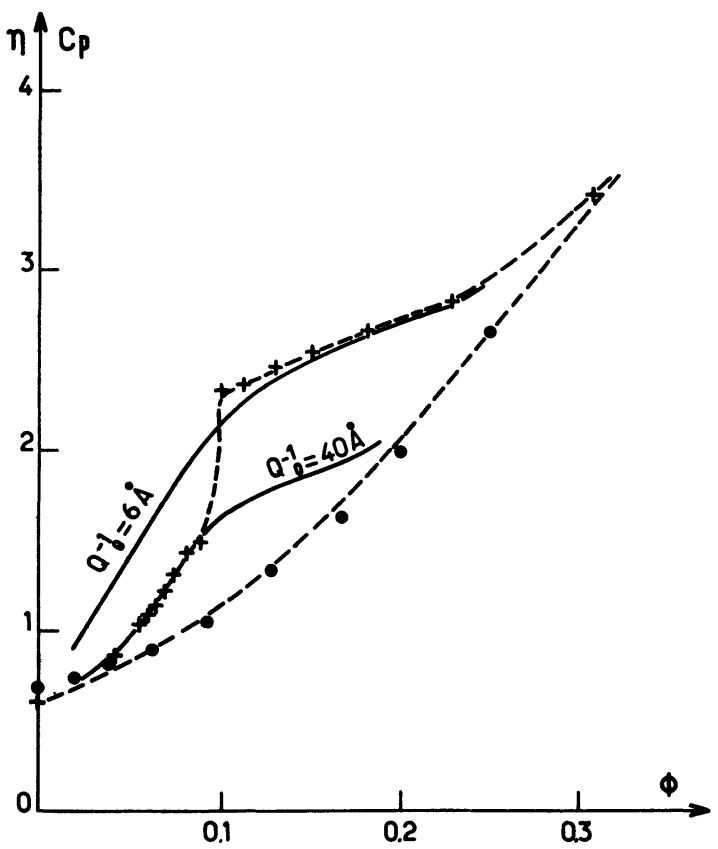

Fig. 5. - Tentative fit of the viscosity data with equation (5). The lowest dotted line represents our choice for $\eta_{\text {reg }}$, the upper dotted line is a guide for the eye. The two theoretical extreme lines correspond to $x_{\eta}=0.15$ and respectively $Q_{0}^{-1}=6 \AA$ and $Q_{0}^{-1}=40 \AA$. Circles and crosses are experimental points.

the two cases a value of $x_{\eta}$ larger than the theoretical values has been found to better represent the data : $x_{\eta}=0.15$. The largest value of $Q_{0}^{-1}$ is $40 \AA$ larger than the typical values of $\xi_{0}$ in microemulsion systems : $\xi_{0} \sim 4-6 \AA[8-10]$. This has already been found in binary mixtures $Q_{0} \xi_{0} \sim 0.3$ [21]. Finally it can be noted that our attempts to fit viscosity data with smaller values of $x_{\eta}$ or an unique value of $Q_{0}$ by changing the background term $\eta_{\text {reg }}$ led in all cases to anomalous regular parts. 
5. Conclusion. - We have observed an anomalous behaviour of the shear viscosity close to a critical point in microemulsion systems. Although there is a good qualitative agreement between experiments and theory, the quantitative comparison is less satisfactory.

Further experimental work is in progress in order to clarify this point. We are trying in particular to approach the critical point more closely in the quaternary toluene-butanol-SDS-water systems. We also plan to study in detail other five component systems (toluene-butanol-SDSwater- $\mathrm{NaCl}$ ) where we have previously observed viscosity anomalies in the microemulsion phases close to critical end points [22], and where plots of the viscosity versus droplets volume fraction reveal anomalies similar to those of figure 2 [23].

\section{References}

[1] Sengers, J. V., in Proceedings of the International School Enrico Fermi, ed. M. S. Green (Acad. Press) 1971.

[2] LeE, S. P., Chem. Phys. Lett. 57 (1978) 611.

[3] Fixman, L., J. Chem. Phys. 36 (1962) 310.

[4] Hohenberg, P. C., Halperin, B. I., Rev. Mod. Phys. 49 (1977) 435.

[5] Ishimoto, C., Tanaka, T., Phys. Rev. Lett. 39 (1977) 474.

[6] Corti, M., Degiorgio, V., Phys. Rev. Lett. 45 (1980) 1045.

[7] Cazabat, A. M., Chatenay, D., Langevin, D., Pouchelon, A., J. Physique-Lett. 41 (1980) L-441.

[8] Cazabat, A. M., Langevin, D., Meunier, J., Pouchelon, A., J. Physique-Lett. 43 (1982) L-89.

[9] Huang, J. S., KIM, M. W., Phys. Rev. Lett. 47 (1981) 1962.

[10] Dorshow, R., De Buzzaccarini, F., Bunton, C. A., Nicoli, D. F., Phys. Rev. Lett. 47 (1981) 1336.

[11] Fourche, G., BellocQ, A. M., Brunetti, S., to be published.

[12] Microemulsions, theory and Practice, ed. by L. M. Prince (Acad. Press) 1977.

[13] Stanley, E., Introduction to phase transitions and critical phenomena (Oxf. Univ. Press) 1971.

[14] Griffiths, R. B., Wheeler, J. C., Phys. Rev. A 2 (1970) 1047.

[15] Deutch, J. M., Zwanzig, R., J. Chem. Phys. 46 (1967) 1612.

[16] OHTa, T., J. Phys. C 10 (1977) 791.

[17] Calmettes, P., Phys. Rev. Lett. 39 (1977) 1151.

[18] Beysens, D., Chen, S. H., Chabrat, J. P., Letamendia, L., Rouch, J., Vaucamps, C., J. Physique-Lett. 38 (1977) L-203.

[19] Belloce, A. M., private communication.

[20] Dvolaitzki, M., Ober, R., Billard, J., Taupin, C., Charvolin, J., Hendricks, Y., C. R. Hebd. Séan. Acad. Sci. Paris 292 II (1981) 45.

[21] Burstyn, H. C., Sengers, J. V., Phys. Rev. A 25 (1982) 448.

[22] Pouchelon, A., Meunier, J., Langevin, D., Cazabat, A. M., J. Physique-Lett. 41 (1980) L-239.

[23] Guering, P., Cazabat, A. M., unpublished results. 